\title{
Activation of the type 3 secretion system of enteropathogenic $E$. coli leads to remodeling of
} its membrane composition

Anish Zacharia ${ }^{1}$, Ritesh Ranjan $\mathrm{Pal}^{2,3}$, Naama Katsowich ${ }^{2}$, Chanchal Thomas Mannully ${ }^{1}$, Aida

ibrahim ${ }^{1}$, Sivan Alfandary ${ }^{1}$, Raphael Serruya ${ }^{1}$, Amit K Baidya ${ }^{2,}{ }^{4}$, Sigal Ben-Yehuda ${ }^{2}$, Ilan Rosenshine $^{2}$, Arieh Moussaieff ${ }^{1} *$

1. The Institute for Drug Research, The Hebrew University of Jerusalem, Jerusalem, Israel

2. Department of Microbiology and Molecular Genetics, Institute of Medical Research IsraelCanada, Faculty of Medicine, The Hebrew University of Jerusalem, Jerusalem, Israel

3. School of Biological Sciences, Indian Association for the Cultivation of Science, Kolkata, India

4. Molecular Biophysics Unit, Indian Institute of Science, Bangalore, India

* For correspondence: ariehm@ekmd.huji.ac.il 
Abstract The type III secretion system (T3SS) is critical for the virulence of enteropathogenic $E$. coli (EPEC), an important human pathogen. T3SS is activated upon host attachment, leading to effector injection, which is associated with repression of CsrA. CsrA repression results in gene expression remodeling, which contributes to EPEC adaptation to a cell-adherent lifestyle. How these changes in gene expression influence EPEC physiology and virulence remains poorly understood. We reasoned that this influence may be mediated by bacterial membrane, and that aside from its well documented influence on central carbon metbaolism, CsrA regulation also confers changes in membrane lipid metabolism. By combining lipidomics with genetic analyses of mutant strains that simulate T3SS activation, we show that activation of the EPEC T3SS is associated with remodeling of lipid metabolism, and in particular, a shift from phospholipids towards lysophospholipids, and from menaquinones and ubiquinones to undecaprenyl lipids. Concomitant to the remodeling of membrane lipid composition, we found higher O-antigen levels, as well as increased cell size and negative cell surface charge in the mutant strains. The shifts we found in membrane lipid compositoion and in O-antigen content are expected to cause changes in memebrane permeability and function. In line with this notion, we found increased sensitivity to vancomycin in the mutant strains.

Importance. EPEC is an important human pathogen. The characterization of its membrane lipid composition upon attachment to host is an important step towards understanding the biology of EPEC and other pathogenic bacteria. We predict that the lipid remodeling upon attachment to host cells and T3SS activation contributes to bacterial fitness and promotes host colonization. 


\section{Introduction}

Enteropathogenic E. coli (EPEC) is a common cause of pediatric diarrhea (1). Upon attachment to the host intestinal epithelium, this pathogen employs a type 3 secretion system (T3SS) to inject effector proteins into the host cells. The T3SS is encoded within a pathogenicity island termed the locus of enterocyte effacement (LEE) (2), composed of a cluster of transcriptional units containing 41 genes encoding for T3SS structural components, six translocated effectors, and related proteins $(3,4)$. The LEE5 operon contains three genes: tir, cesT, and eae, encoding Tir (Translocated intimin receptor), CesT, and intimin, respectively $(5,6)$. Tir is the most abundant effector and the first to be translocated into the host cell $(7,8)$. CesT is a homodimer chaperone associated with many effectors, of which Tir is the major one $(9,10)$. CesT binds to two regions in Tir at the $\mathrm{N}$ - and $\mathrm{C}$-terminus through a specific recognition motif, promoting Tir stability and its translocation to the host (9). Immediately following its translocation into the host, Tir is targeted to the host cell membrane and becomes an integral membrane protein with two transmembrane domains and a surface-exposed loop. Intimin, the third product of the LEE5 operon, is an outer membrane protein that promotes adherence of EPEC to the host via interaction with the surface exposed loop of translocated Tir $(11,12)$. This attachment type was termed intimate attachment (11).

CesT-Tir interaction is essential for Tir translocation into the host and the subsequent intimate attachment action. The second function of CesT-Tir interaction is related to the rearrangement of gene expression upon EPEC-host contact and the consequent Tir translocation (13). In planktonic EPEC, CesT remains bound to Tir and other effectors and the T3SS is not active but fully assembled, ready to immediately translate the effectors (14). The "gate-keeper" complex composed of SepL-CesL and SepD prevents secretion of effectors by planktonic EPEC. Upon bacteria-host contact, the gate-keepr no longer inhibits the T3SS and it is activated, followed by immediate and rapid delivery of Tir and other effectors into the host cell $(8,9,14,15)$. The delivery of these effectors into the host liberates CesT resulting in increased levels of free CesT in the EPEC cytoplasm. The liberated CesT then interacts with an alternative binding partner, the carbon storage regulator A (CsrA) $(9,13,16)$. CsrA is an RNA-binding protein and posttranscriptional regulator, which coordinates numerous bacteria functions, including motility, metabolism, and virulence $(17,18)$. Notably, the elevated levels of free CesT, upon effectors injection, competitively inhibit CsrA-mRNA interaction $(9,13,16)$. Since CsrA binds to the 
bioRxiv preprint doi: https://doi.org/10.1101/2020.12.17.423234; this version posted June 27, 2021. The copyright holder for this preprint (which was not certified by peer review) is the author/funder, who has granted bioRxiv a license to display the preprint in perpetuity. It is made available under aCC-BY-NC 4.0 International license.

mRNA of numerous genes and regulates the stability and/or translation of these mRNAs, CesTCsrA interaction and the consequential liberation of the mRNAs result in remodeling of gene expression (Fig. 1).

(A)
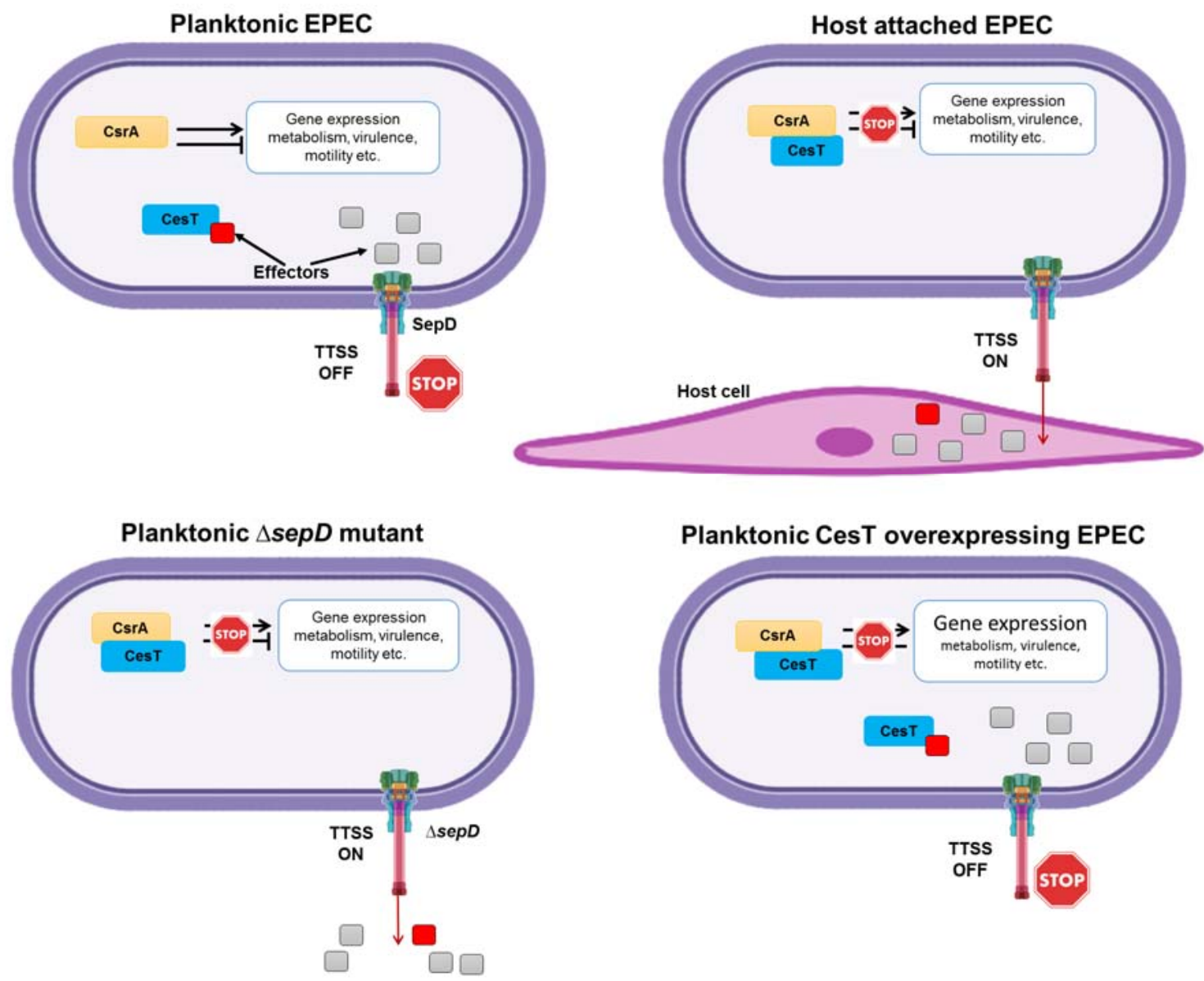

Fig 1 A scheme of the T3SS regulation of EPEC attachment to the host cell.

(A) Planktonic EPEC: The T3SS is not active. The effectors accumulate in the cytoplasm, and some of them (indicated as a red square) bind to CesT and sequester it. In these bacteria, CsrA is free to bind mRNA and regulate (mostly repress) gene expression.

(B) Host-attached EPEC: The T3SS is activated upon contact with the host, eliminating the effectors from the EPEC cytoplasm by injecting them into the host cell. The liberated CesT then binds to CsrA to inhibit CsrA-mRNA interaction.

(C) Planktonic $\triangle s e p D$ mutant: The T3SS is constitutively active, and effectors are secreted. The liberated CesT binds to CsrA and inhibits CsrA-mRNA interaction.

(D) Planktonic CesT overexpressing EPEC: CesT is expressed at levels that allow binding to all the CesT-binding effectors and CsrA, resulting in inhibition of CsrA-mRNA interactions regardless of T3SS activity. 
The significance of the CesT-CsrA switch to the bacterial physiology can be partially extrapolated from studies comparing wild type E. coli to the $\operatorname{csr} A$ mutant with null or residual activity $(17,19$ 22). However, $\operatorname{csr} A$ mutants are not the ideal model to mimic host interacting EPEC since the CesT-CsrA interaction induces only partial and temporal CsrA inhibition. Previous reports point to alternative approaches that mimic the physiological state of host-attached EPEC. The first approach employs the EPEC sepD mutant $(\triangle \operatorname{sep} D)$, which expresses T3SS that constitutively secretes Tir regardless of host attachment $(13,23)$. Thus, the sepD mutant contains higher levels of liberated CesT that is free to interact with CsrA. Another approach uses wild-type EPEC containing a plasmid that expresses CesT under an isopropyl-D-thiogalactopyranoside (IPTG) regulated promoter $(\mathrm{EPEC} / \mathrm{pCesT})$. Upon IPTG treatment it overexpresses CesT that readily interacts with CsrA (Fig. 1).

Here, we combined lipidomics and genetic methodologies to characterize the shift of EPEC metabolism under conditions that mimic attachment to the host cell and the associated CsrA inhibition. Surprisingly, our lipidomics analysis pointed to a major metabolic signature of this shift. We expect this lipid shift to be a universal trait in the E. coli spp. when experiencing CsrA inhibition. We further assume that this lipid shift is involved in the adaptation of EPEC to a celladherent lifestyle.

\section{Results}

We sought to unveil possible shifts in membrane lipid metabolism that take place in EPEC upon T3SS activation and effector injection, by an unbiased lipidomics analysis. However, obtaining biological material from injecting EPEC in quantities that allow lipidomics analysis is challanging, as one small subpopulation is engaged in injection, while in the other subpopulation the T3SS remains inactive (8). To overcome this hurdle and obtain uniform populations, we compared wild type EPEC that does not secrete Tir (thus Tir sequesters CesT), to an isogenic EPEC $\triangle$ sepD mutant that constitutively secretes Tir, and consequently, CesT is liberated, free to interact with CsrA. To verify the CsrA-mediated regulation of lipid metabolism, we used EPEC with a deleted $C s r A$ gene $(\Delta c s r A)$. The latter was also used as a reference to available data of the 
metabolic profile of the EPEC and E. coli K12 $\Delta$ csrA mutant $(21,24,25)$. We grew the bacteria under conditions that promote the expression of the T3SS genes (i.e., growth in $500 \mathrm{~mL}$ DMEM, $37^{\circ} \mathrm{C}$, without shaking to $\left.\mathrm{OD}_{600} 0.6\right)$. Under our experimental conditions, $\Delta c s r A$ strains showed attenuated growth rate (Fig. S1), in line with previous literature $(18,21,25)$. The $\Delta$ sepD $\Delta$ cesT double mutant showed a mild attenuation in growth rate, wheras the $\Delta s e p D$ strain and the pCesT strains showed similar growth rates to the wild type.

\section{CesT-CsrA interaction confers alterations in phospholipid and terpenoid-quinones pathways}

To have a global and unbiased evaluation of the influence of the attachement to host followed by CsrA inhibition on bacteria membrane, we performed a lipidomics study. To mimic the attachement to host cells we studied the lipid composition of wild type EPEC, $\triangle$ sepD mutant, and wild type EPEC overexpressing recombinant CesT from a plasmid (EPEC/pCesT). Bacteria of the latter strain transiently, or partially, inhibit CsrA (as in attached EPEC). A total of 15,827 mass features were detected in a dataset that included all the strains. After the exclusion of possible artifact features (see Methods section), analysis was carried out using 3,277 mass features. We first analyzed the lipid composition of wild type vs the sepD mutant. Principal component analysis (PCA) demonstrated a striking separation between the lipidome of the $\Delta$ sepD mutant and that of wild type EPEC, with PC1 accounting for $97.9 \%$ of the variance (Fig. 2A), suggesting that the increased levels of free CesT in the $\triangle \operatorname{sepD}$ mutant induced a considerable shift in lipid metabolism. To test this hypothesis, we compared the lipidome of both strains to that of a mutant with both sepD and cesT deleted ( $\triangle \operatorname{sepD} \Delta \mathrm{ces} T)$. Indeed, the PCA of the lipidome of the $\Delta s e p D$ $\Delta c e s T$ examined showed no shift in the lipid composition compared to the wild type strain in the absence of CesT (Fig. 2B). To further confirm that the increased levels of free CesT are responsible for the lipid shift, we overexpressed free CesT in wild type EPEC (EPEC/pCesT). The overexpression of CesT was sufficient for inducing a shift in lipid composition (Fig. 2B). As can be expected, the change in lipid composition, seen in principal component 1 of the PCA is increased in EPEC/pCesT, likely due to higher levels of CesT. Taken together, our data suggest that an increase in the levels of CesT is necessary and sufficient to induce a considerable shift in the lipid composition of EPEC. A Variable Importance in Projection (VIP) analysis of $\triangle \operatorname{sepD}$ vs. 
wild type EPEC lipidome revealed 54 differential (VIP>1) mass features. PLs and isoprenoids of the quinone terpenoid subclass were predominant in this analysis (Fig. 2C). Within the differential lipids, all 4 lysophospholipids (LysoPLs) were highly accumulated in the SepD mutants. The distribution of PLs in the differential lipids was more complex. All 6 upregulated PLs were phosphatidylethanolamines (PEs), while out of the 9 PLs in the group of lipids that were downregulated in SepD mutants 6 were PEs, 3 phosphatidylglycerols (PGs) and 2 phosphatidylserine (PS). All menaquinone and ubiquinone were downregulated in the SepD mutants. We therefore focused our analyses thereof on the terpenoid-quinones isoprenoid and phospholipid pathways, and identified lipids of the two main differential classes from the whole dataset.

(A)

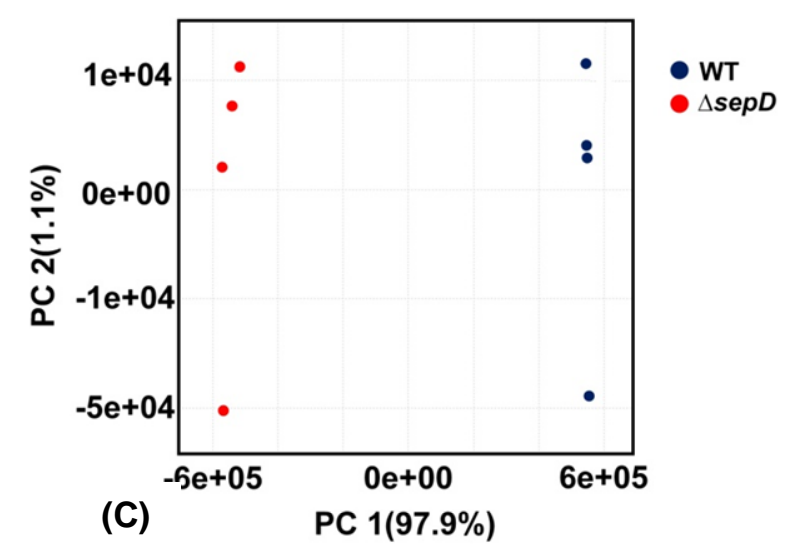

(B)

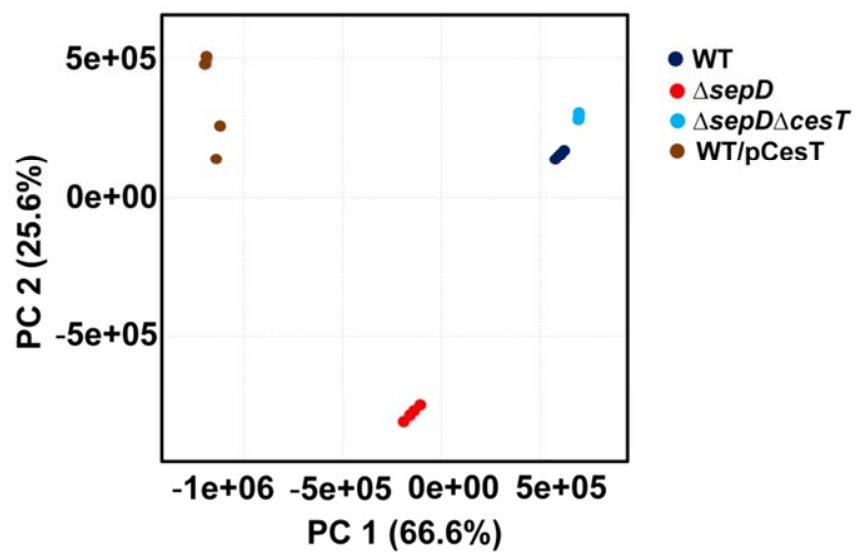

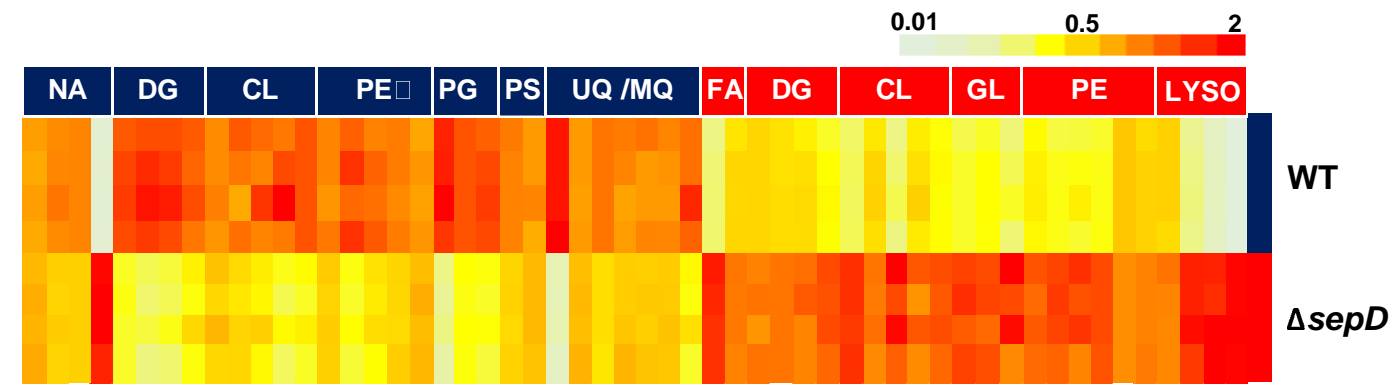

Fig 2 T3SS exerts a profound effect on EPEC lipid metabolism. (A) Principal component analysis (PCA) of lipids in sepD mutant EPEC vs. wild type strain (WT) $(\mathrm{N}=4)$. (B) PCA of the lipid compositon of WT, $\Delta s e p D, \Delta s e p D \Delta c e s T$ and pCesT. (C) A heat map 
of the differential lipids (Variable Importance in Projection (VIP) $>1$ ) in $\operatorname{sep} D$ mutants divided into lipid subclasses. Lipid subclasses with upregulated accumulation in mutants are in red and lipid subclasses with downregulated accumulation in mutants are in blue quadrants. Colour in each column indicates the fold change from mean abundance of the particular feature across all samples. Lyso, lysophospholipids; GL, Glycolipids; CL, Cardiolipins; DG, Diglyceride/Diacylglycerol; FA, Fatty acids; Q/MK, Ubiquinone/Menaquinone; PE, phosphatidylethanolamines; PG, phosphatidylglycerols; PS, phosphatidylserines; NA, not assigned.

\section{T3SS activation drives a remodeling of the glycerophospholipid pathway}

Our VIP analysis pointed to phospholipids and quinone terpenoids as major differential groups of lipids upon T3SS activation, and suggested a metabolic shift from PGs in the glycerophospholipid pathway. To better define the shifts suggested by the VIP analysis, we quantified the abundance of all identified lipid species per subclasses. We first quantified the major membrane constituents PEs (total abundance of 29 identified lipid species) and PGs (total abundance of 31 identified lipid species) in the different strains. In line with the VIP analysis, we found a striking decrease in the abundance of PGs in $\triangle s e p D$ and pCesT EPEC (Fig. 3A), whereas mild increase in that of PEs in $\triangle s e p D$ EPEC (Fig. 3B). Also, aligned with our VIP analysis, we found a higher abundance of the identified LysoPLs (total abundance of 24 identified lipid species; (Fig. 3C)). We identified lysophosphatidic acids (LysoPAs; total abundance of 9 identified lipid species; (Fig. 3D)), dramatically more abundant in $\triangle s e p D$ and pCesT EPEC. Cardiolipins (CLs; total abundance of 20 identified lipid species; (Fig. 3E)) were upregulated in the mutant strain as well.

To get a complete picture of the T3SS-dependent lipid metabolism in E coli, we mined a CsrAmutant transcriptome database (24) for the glycerophospholipid pathway genes. We integrated our lipidomics data and the published gene expression (RNA-seq) data for a better understanding of the PL network. The gene expression data was well-aligned with the results of our analyses of the abundance of PL subclasses (Fig. S2), although EPEC were cultivated under different conditions. Altogether, our data on PL metabolism suggest a shift from of PGs, towards PEs and LysoPLs on the one hand, and on the other hand, towards CLs . 
(A)

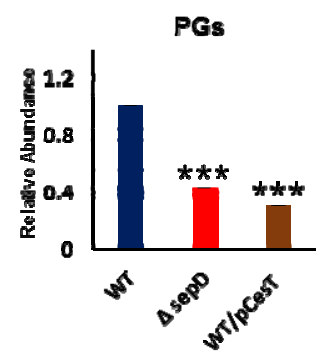

(B)

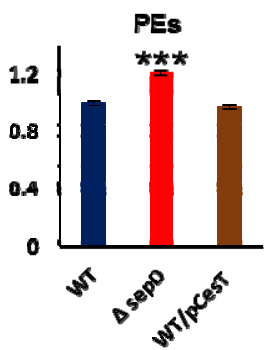

(C)

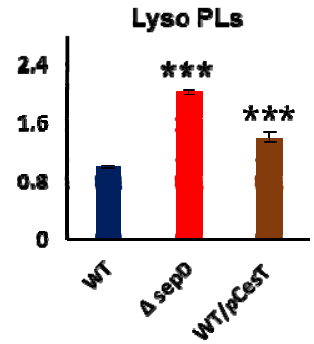

(D)
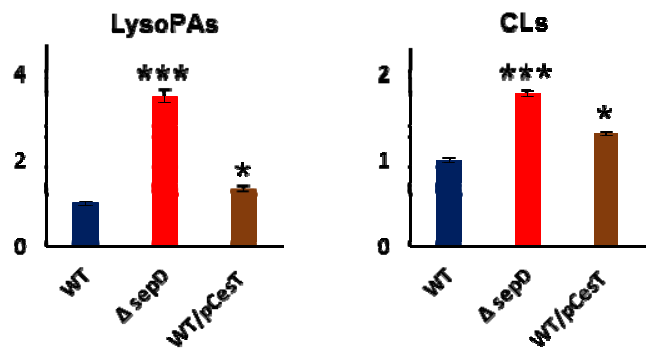

(F) Glycerophospholipid Pathway

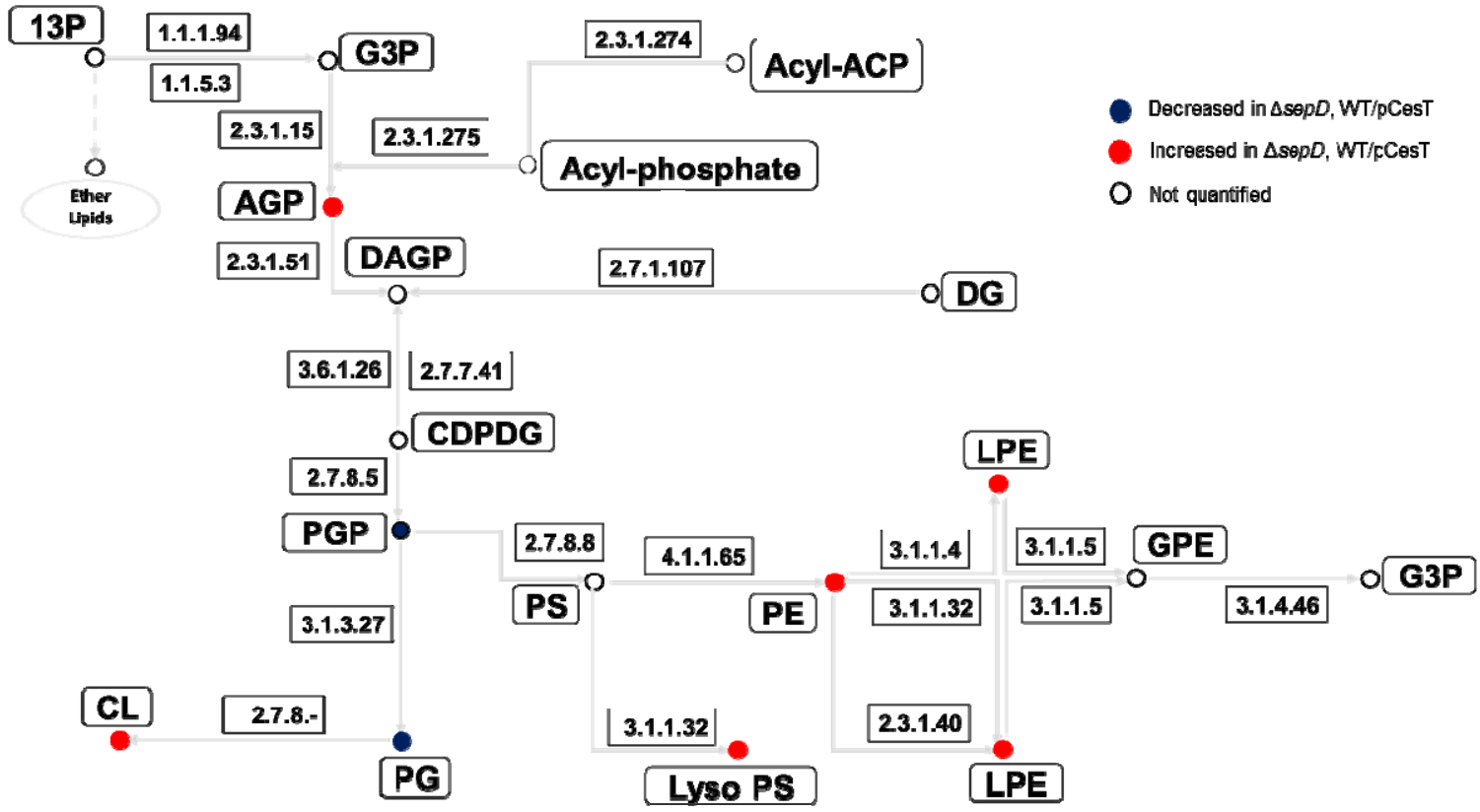

Fig 3 Inhibition of CsrA by CesT leads to a shift from phosphatidylglycerols (PGs) to phosphatidylethanolamines (PEs) and lysophospholipids (LysoPLs) on the one hand and CLs on the other. (A) The relative total abundance of identified PGs (31 identified lipid species; A); PEs (29 identified lipid species; B); LysoPLs (24 identified lipid species; C), lysophosphatidic acids (9 identified lipid species; LysoPAs; D) and cardiolipins (20 identified lipid species; CLs; E) is presented. (D) A metabolic map of phospholipids with our lipidomics data. Lipid classes are represented by circles, with their names in white quadrants. Lipids abbreviations: 13P, Glycerone phosphate; G3P, Glycerol-3-phosphate; AGP, Acylglycerol-3-phosphate; DAGP, 1,2-Diacylglycerol 3-phosphate; CDPDG, 1,2-Diacylglycerol-cytidine 5-diphosphate; GPE, Glycerophosphoethanolamine; LPE, Lysophosophoethanolamine; Lyso PS, Lysophosphoserine;PGP, phosphatidylglycerophosphate; PE, Phosphatidylethanolamine; PS, Phosphatidylserine; PG, Phosphatidylglycerol. Enzymes are represented by arrows, with their numbers in quadrants. Enzymes common nomenclature is used in the scheme. For an unambiguous identification, enzyme nomenclature (EC number system) is hereby given, along with further commonly used names: [1.1.1.94]- gpsA, glycerol-3-phosphate dehydrogenase; [1.1.5.3]- glpD, sn-glycerol-3-phosphate 
dehydrogenase; [2.3.1.15]- plsB, glycerol-3-phosphate O-acyltransferase; [2.3.1.274]- plsX, phosphate acyltransferase; [2.3.1.275]plsY (ygiH), glycerol-3-phosphate acyltransferase; [2.3.1.51]- plsC (parF), 1-acyl-sn-glycerol-3phosphate acyltransferase; [2.7.1.107]- dgkA, diacylglycerol kinase; [3.6.1.26]- cdh, CDP-diacylglycerol phosphotidylhydrolase; [2.7.7.41]- cdsA, (cds) CDPdiglyceride synthase; [EC:2.7.8.5]- pgsA, phosphatidylglycerophosphate synthetase; [3.1.3.27]- pgpA (yajN), phosphatidylglycerophosphatase A; [2.7.8.8]- pssA (pss), phosphatidylserine synthase; [3.1.1.32]- pldA, outer membrane phospholipase A; [4.1.1.65]- psd, phosphatidylserine decarboxylase; [3.1.1.4]- pldA, outer membrane phospholipase A; [2.3.1.40]aas, acyl-acyl carrier protein synthetase; [3.1.1.5]- pldB, lysophospholipase L; [3.1.4.46]- glpQ (ugpQ), glycerophosphodiester phosphodiesterase. Data are presented as mean $\pm \mathrm{SE}(\mathrm{N}=4)$. *, $\mathrm{p}<0.05 ; * * *, \mathrm{p}<0.001$.

\section{Undecaprenyl lipid biosynthesis is upregulated, whereas synthesis of menaquinones and ubiquinones is downregulated upon T3SS activation.}

Our analysis of the differential lipids (Fig. 2C) suggests that isoprenoid terpenoids biosynthesis is also altered in sepD mutants. Accordingly, we compared the levels of identified isoprenoid species per subclasses in the $\triangle s e p D$ EPEC to those of a wild type strain. To corroborate the T3SSdependent lipid profile, to exclude possible non-specific effects of the mutation, and to suggest a mechanistic insight into the SepD-dependent lipid regulation, we studied the lipid composition upon CesT overexpression (EPEC/pCesT). This analysis pointed to increased accumulation of endproducts of the undecaprenyl, while decreased ubiquinone and menaquinone endproducts accumulation, and suggested a shift towards undecaprenyl biosynthesis in the sepD mutants (Fig. 4A-D). Notably, this shift was recapitulated and was, in fact, more pronounced in EPEC overexpressing CesT (Fig. 4A-D). 
bioRxiv preprint doi: https://doi.org/10.1101/2020.12.17.423234; this version posted June 27, 2021. The copyright holder for this preprint (which was not certified by peer review) is the author/funder, who has granted bioRxiv a license to display the preprint in perpetuity. It is made available under aCC-BY-NC 4.0 International license.

(A)

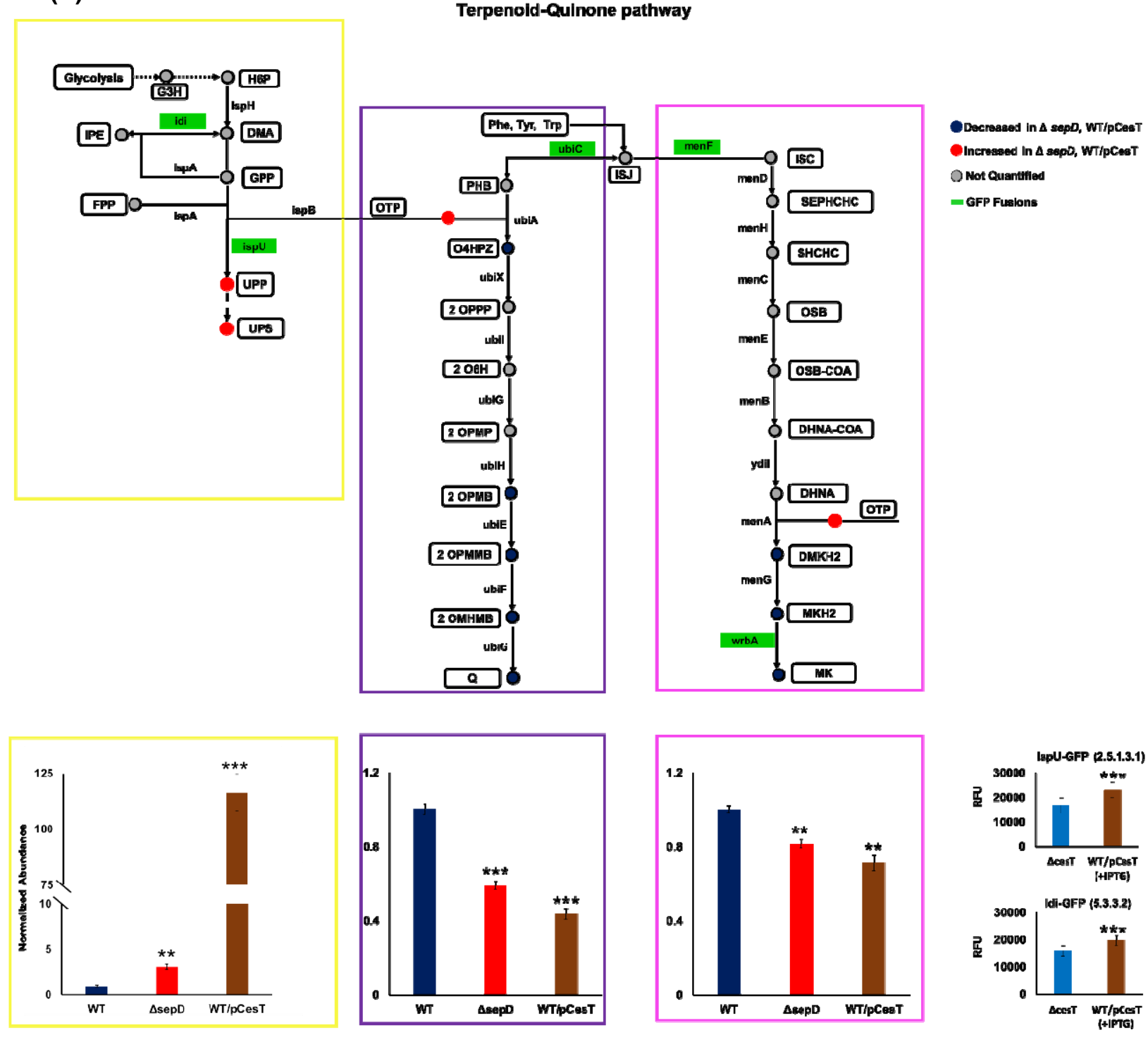

Fig 4 Activation of T3SS in EPEC upregulates the biosynthesis of undecaprenyl lipids and downregulates the biosynthesis of menaquinones and ubiquinones. (A) Terpenoid-quinones network was adapted from Kyoto Encyclopedia of Genes and Genomes (KEGG) for Escherichia coli O127:H6 E2348/69 (EPEC), and modified to include the undecaprenyl, ubiquinone and menaquinone branches of terpenoid pathway, resulting in a simplified scheme of the metabolic network of terpenoid quinone lipids, divided into (A) undecaprenyl (yellow box), (B)ubiquinone (purple box), and (C) menaquinone (pink box) lipids. The colors of lipid species (in circles) represent their relative accumulation according to our analyses. Lipid species and subclasses are represented in white boxes and green boxes represent enzyems fused to GFP; their expression was determined in terms of relative fluorescence unit (RFU). (D) Accumulated abundance of undecaprenyl lipids, ubiquinones, and menaquinones, respectively, in 'host cell attachment' like conditions. (E) GFP flourescence was measured in the strains with null cesT and pCesT. Lipids abbreviations: H6P, 1-Hydroxy-2methyl-2-butenyl- 4-diphosphate; DMA, Dimethylallyl diphosphate; IPE, Isopentenyl diphosphate; GPP, Geranyl diphosphate; FPP, Farnesyl diphosphate; UPP, Undecaprenyl diphosphate; UPS, undecaprenyl species OTP, Octaprenyldiphosphate; PHB, Hydroxybenzoic acid; ISJ, Chorismate; O4HPZ, 4-Hydroxy-3-polyprenyl benzoate; 2OPPP, 2-Octaprenyl phenol; 2O6H, 2Octaprenyl-6-hydroxyphenol; 2OPMP, 2-Octaprenyl-6-methoxy phenol; 2OPMB, 2-Octaprenyl-6-methoxy-1,4-benzoquinone; 2OPMMB, 2-Octaprenyl-3-methyl-6-methoxy-1,4-benzoquinone; 2OMHMB, 2-Octaprenyl-3-methyl-5-hydroxy-6-methoxy-1,4benzoquinone; Q, Ubiquinone; ISC, Isochorismate; SEPHCHC, 2-Succinyl-5-enolpyruvyl-6-hydroxy-3-cyclohexene-1carboxylate; SHCHC, 6-Hydroxy-2-succinylcyclohexa-2,4-diene-1-carboxylate; OSB, 2-Succinylbenzoate; OSB-COA, 2Succinylbenzoyl-CoA; DHNA-CoA, 1,4-Dihydroxy-2-naphthoyl-CoA; DHNA， 1,4-Dihydroxy-2-naphthoate; DMKH2, 
Demethylmenaquinol; MKH2, Menaquinol; MK, Menaquinone. Enzymes are represented by arrows, with their numbers in quadrants. Enzymes common nomenclature is used in the scheme. For an unambiguous identification, enzyme nomenclature (EC number system) is hereby given, along with further commonly used names: [1.17.7.4]- ispH (yaaE, lytB), 4-hydroxy-3-methylbut2-enyl diphosphate reductase; [2.5.1.1, 2.5.1.10]- ispA, farnesyl diphosphate synthase; [2.5.1.90]- ispB (cel, yhbD), all-transoctaprenyl-diphosphate synthase; [2.5.1.31]- ispU (uppS, rth, yaeS), ditrans,polycis-undecaprenyl-diphosphate synthase; [4.1.3.40]- ubiC, chorismate lyase; [2.5.1.39]- ubiA, 4-hydroxybenzoate octaprenyltransferase; [2.5.1.129]- ubiX (dedF), 3octaprenyl-4-hydroxybenzoate carboxy-lyase; [1.14.13.240]- ubiI (visC), 2-methoxy-6-(all-trans-octaprenyl)phenol 4-hydroxylase; [2.1.1.222, 2.1.1.64]- ubiG (pufX, yfaB), bifunctional 3-demethylubiquinone-8 3-O-methyltransferase and 2-octaprenyl-6hydroxyphenolmethylase; [1.14.13.-]- ubiH (acd, visB) 2-octaprenyl-6-methoxyphenol 4-hydroxylase; [2.1.1.201]- ubiE (yigO), bifunctional 2-octaprenyl-6-methoxy-1,4-benzoquinol methylase and demethylmenaquinone methyltransferase; [1.14.99.60]- ubiF (yleB), 2-octaprenyl-3-methyl-6-methoxy-1,4-benzoquinol oxygenase, 2-octaprenyl-3-methyl-6-methoxy-1,4-benzoquinol hydroxylase; [2.1.1.222, 2.1.1.64]- ubiG (pufX, yfaB) bifunctional 3-demethylubiquinone-8 3-O-methyltransferase and 2octaprenyl-6-hydroxyphenol methylase; [5.4.4.2]- menF (yfbA), isochorismate synthase 2; [2.2.1.9]- menD, 2-succinyl-5enolpyruvyl-6-hydroxy-3-cyclohexene-1-carboxylatesynthase; [4.2.99.20]- menH (yfbB), 2-succinyl-6-hydroxy-2,4cyclohexadiene-1-carboxylate synthase; [4.2.1.113]- menC, o-succinylbenzoate synthase; [6.2.1.26]- menE, osuccinylbenzoateCoA ligase; [4.1.3.36]- menB, 1,4-dihydroxy-2-naphthoyl-CoA synthase; [3.1.2.28]- ydiI (menI), 1,4-dihydroxy2-naphthoyl-CoA hydrolase; [2.5.1.74]- menA (yiiW), 1,4-dihydroxy-2-naphthoate octaprenyltransferase; [2.1.1.163, 2.1.1.201]ubiE (menG, yigO), demethylmenaquinone methyltransferase /2-methoxy-6-octaprenyl-1,4-benzoquinol methylase; [1.6.5.2]$\operatorname{wrbA}(\mathrm{ytfG})$, quinone oxidoreductase. Data are presented as mean $\pm \mathrm{SE} . \mathrm{N}=4$. **, $\mathrm{p}<0.01 ; * * *, \mathrm{p}<0.001$.

We then tested the influence of CesT levels on the production of key enzymes involved in the terpenoid-quinones biosynthesis. To this end, we fused the GFP reporter gene in frame to the 3' end of a selected number of genes in their native chromosomal location without perturbing their tarnscritional unit. Thus, the GFP levels indicate both efficiency of enzyme production and stability. We selected for tagging genes whose GFP-tagging was previously found to be tolerated by the bacteria (26). Using this approach, we GFP-tagged the following genes in wild type EPEC or the $\Delta c e s T$ mutant: $i d i$, which forms isopentenyl pyrophosphate, an isoprenoid precursor; ispU, involved in the biosynthesis of undecaprenyl pyrophosphate; $u b i C$ and menF, bridging between menaquinone and ubiquinone pathways, and $w r b A$ encoding for the menaquinone enzyme. We then fluorometrically compared the GFP levels in bacteria lacking and overexpressing CesT in these strains. We detected upregulation of Idi-GFP and IspU-GFP levels upon CesT overexpression (Fig. 4E). This increase is in agreement with the lipid accumulation that we measured. Changes in the levels of GFP-tagged enzymes involved in menaquinone and ubiquinone production were not significant (Fig. S3). Our lipidomics analysis and the quantification of the expression of GFP-tagged idi and IspU are aligned with the data we mined on the expression of genes of the quinone terpenoids pathways in CsrA null EPEC (Fig. S4).

Our analyses point to a metabolic shift towards undecaprenyl species upon T3SS activaiton. Importantly, undecaprenylpyrophoshate (UPP) is required for the synthesis of O-antigen repeating units on surface of the inner leaflet of the inner membrane, and for the flipping of the UPP-O- 
antigen complex to the outer liflet, where it is used as a precursor for the biosynthesis of the LPS $\mathrm{O}$-antigen and $\mathrm{O}$-antigen capsule. We expected the increase in the level of undecaprenyl species we found in SepD mutants to be reflected in higher levels of O-antigen presented on the bacteria cell surface. To define possible alterations in O-antigen levels, we carried out western blot analysis of O-Antigen repeats, which suggested increased levels of LPS O-antigen in the $\Delta s e p D$ mutant and CesT overexpressing EPEC strains (Fig. 5A). An immunofluorescence microscopy demonstrated increased levels of O-antigen (LPS and/or capsular) on the surface of $\triangle s e p D$ mutant and CesT overexpressing EPEC strains, (Fig. 5B-C), in corcordance with the total O-antigen levels observed in western blot analysis.
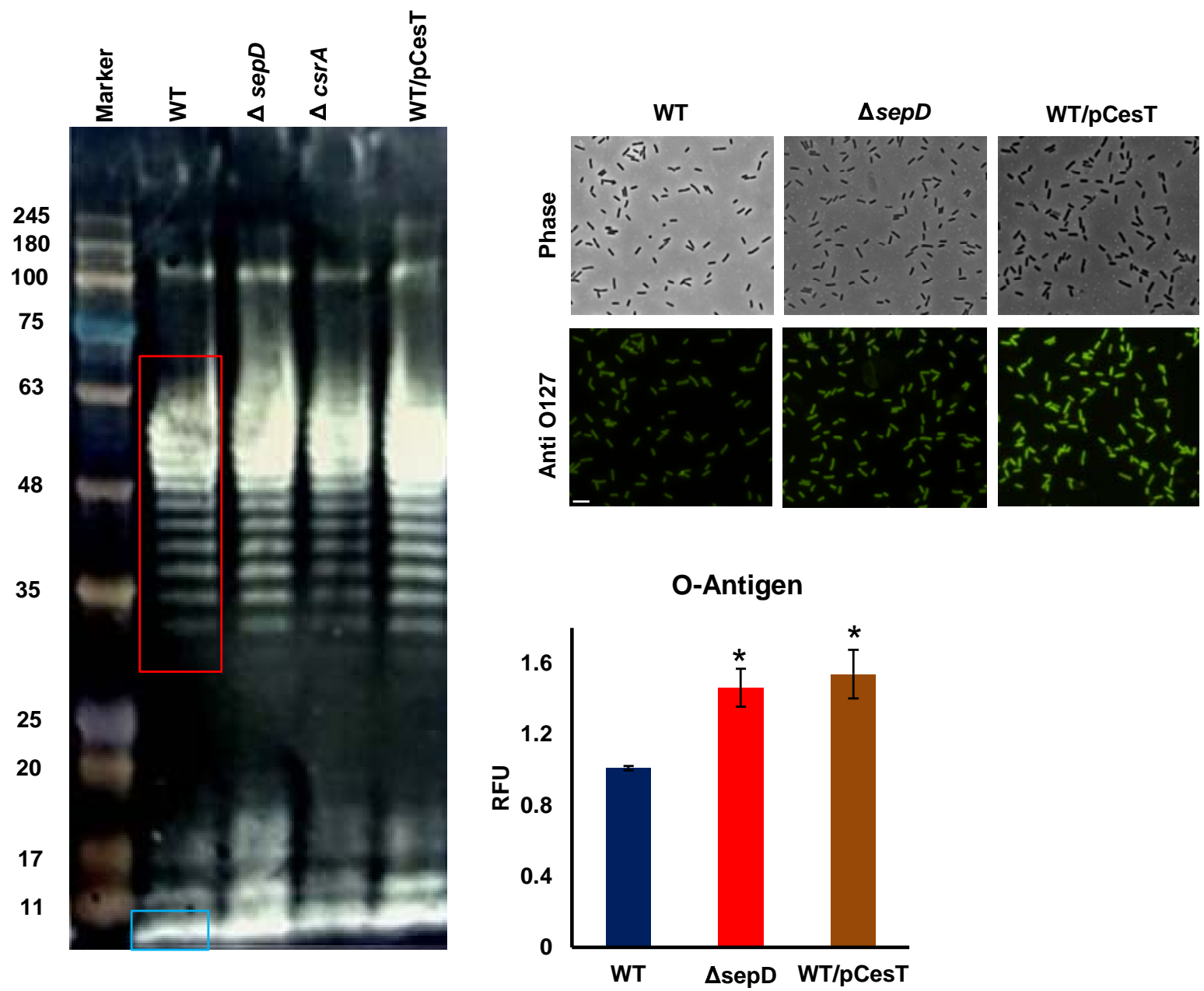

Fig 5 O-Antigen levels in EPEC increase upon T3SS activation. Indicated EPEC strains were subcultured in DMEM at $37^{\circ} \mathrm{C}$ to an $\mathrm{OD}_{600}$ of 0.6. (A) LPS was extracted and subjected to western blotting. O-antigen repeats are boxed in red and the core is in blue 
box. (B-C) Bacteria were fixed, incubated with anti-O127 antibody, and visualized under phase contrast and flurescent optics, and the relative fluorescence determined. Data are presented as mean \pm SE. $N=3$. *, $p<0.05$.

We reasoned that the changes found in the composition of EPEC membrane lipids may be reflected in its physiological and morphological characteristics, such as cell size and cell surface area/volume ratio. Therefore, we measured the cell size of $\triangle \operatorname{sepD}$ mutant and EPEC/pCesT and found significant increases in their size (Fig. 6A), consistant with previous observation made in CsrA mutant E coli (18). Surprisigly, however, the size of the csrA mutant was similar to that of wild type EPEC. The O-antigen content and composition may influence cell surface charge, which plays a role in adhesion of bacteria to oppositely charged surfaces. We thus compared the Zeta potential of wild type EPEC to that of the mutants and found an increase in the negative charge of the mutant strains (Fig. 6B). Given that E. coli is susceptible to vancomycin when cell envelope biogenesis is inhibited (27), we examined EPEC growth inhibition by vancomycin, and found inhibition of growth rates of treated $\Delta s e p D$ and pCesT EPEC (Fig. 6C). To further explore the influence of T3SS on the interactions between the host and bacteria-membrane, we cultured the bacterial strains with diluted serum, and noted a reduced resistance to serum in WT/pcesT and $\triangle$ sepD mutants (Fig. S5). Assuming that the higher permeability to vancomycin may result from the shift in phospholipid metabolism, and concomitant reduction of memberane integrity, we stained the membrane by the liphophilic dye, FM464 that stains membrane lipids and found a lower uptake of FM464 dye uptake with $\Delta s e p D$ and CesT overexpression strains (Fig. S6). 

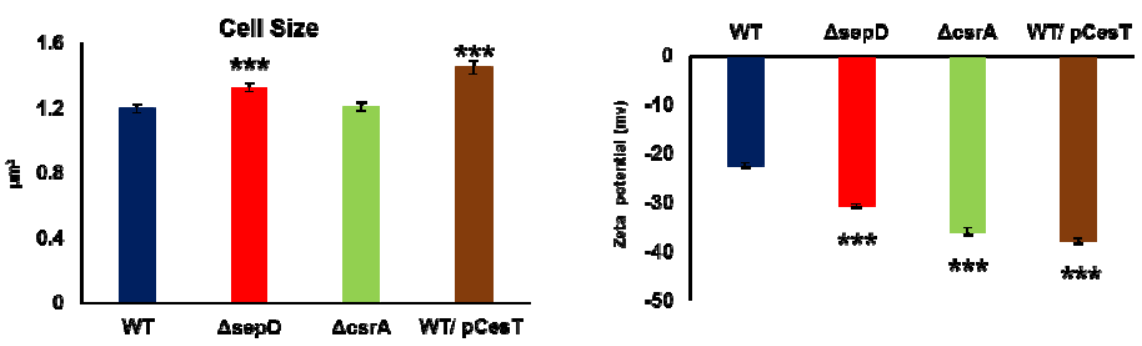

Vancomycin Bactericidal Assay
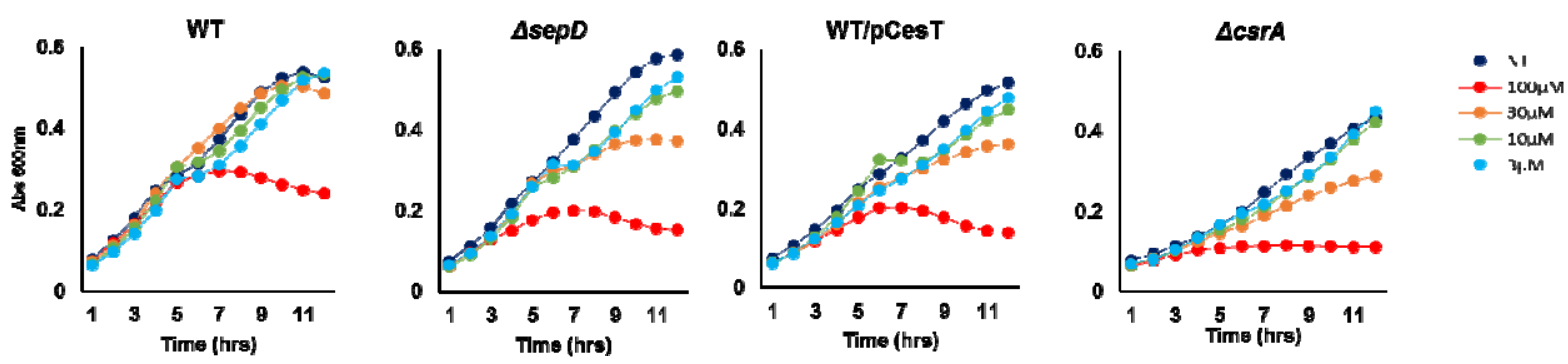

Fig 6 Cell size and effective electric charge on the bacteria's surface increase upon T3SS activation. Wild type (WT), $\triangle$ sepD EPEC strains were subcultured in DMEM at $37^{\circ} \mathrm{C}$ to an $\mathrm{OD}_{600}$ of 0.6 . Cell size (A) of $\sim 150$ bacteria per group was measured under a microscope $(\mathrm{N}=12)$. Measurements were then verified using Zetasizer Nano ZS, by which Zeta potential was measured. For Zeta potential measurements each sample was analyzed in triplicates and the mean charge was recorded (B). The data presented in A-B is the mean $\pm \mathrm{SE}, *, \mathrm{p}<0.05$; ***. (C) The resistance of EPEC strains to vancomycin concnentrations $(3-100 \mu \mathrm{M})$ tested as cell density at $600 \mathrm{~nm}$ throughout 12 hours $(\mathrm{N}=3)$.

\section{Discussion}

Many studies demonstrate the impact of CsrA on E. coli physiology and central carbon metabolism, mostly in the laboratory K12 model strains (17, 19-22), but also in other E. coli isolates (28), including EPEC (24). Our study elucidates a new aspect of the CsrA function that was previously overlooked. We show here the critical impact of T3SS/CesT regulation of CsrA in controlling E. coli lipid composition. Our data suggest that CsrA, in addition to the well documented effects on central carbon metabolism, exerts considerable effects on lipid metabolism and the bacterial envelope structure and function. Linking the changes we found in lipid metabolism to changes in other metabolic networks, and in particular to central carbon metabaolism would be of interest. Importantly, our results point to the T3SS-dependent nature of EPEC lipid metabolism associated with CsrA repression. Most notably, a shift from PGs to PEs and LysoPLs, as well as a shift from menaquinones and ubiquinones towards the production of undecaprenyl lipids. 
An important motivation for carrying out this study was elucidating how the activation of the T3SS, followed by effector injection and intimate attachment to the host, would influence EPEC metabolism. We consider that EPEC $\triangle \operatorname{csr} A$ mutant would only partially phenocopy the physiological state of attached EPEC. This is since this mutant shows reduced growth rate and in addition may collect suppressor mutations. To better mimic T3SS activation upon host attachment, which is followed by a rise in the levels of free CesT and transient CsrA inhibition, we capitalized on engineered EPEC strains that transiently and partially inhibit CsrA (as in attached EPEC) and show a growth rate similar to that of the wild type EPEC in our experimental conditions. These strains include a $\triangle s e p D$ mutant that constitutively secretes Tir and EPEC/pCesT that overexpresses CesT upon induction with IPTG. Using these strains, we carried out a lipidomics study. For a complete picture of the T3SS-dependent lipid metabolism, we mined a csrA-mutant transcriptome database (24). We integrated our lipidomics data and the published gene expression data of EPEC wild type strain E2348/69 and csrA deletion mutant for a better understanding of the shifts in glycerolipid and quinone terpenoids pathways. In a previous analysis by Berndt and colleagues EPEC were cultivated on Modified M9 medium supplement with low glucose $\left(0.02-0.2 \%\right.$ glucose $(\mathrm{w} / \mathrm{v})$ incubated at $\left.37^{\square} \mathrm{C}, 160 \mathrm{rpm}\right)$, and their metabolome examined (24). Our lipidomics analysis points to higher levels of LysoPLs, whereas the data of Berndt et al. disagree with this premise. This discrepancy may reflect differences in experimental conditions used in the two studies. For example, we grew the bacteria statically in DMEM to $\mathrm{OD}_{600}$ 0.6, while Brendt et al. used modified M9 medium and grew the bacteria to $\mathrm{OD}_{600}$ 1.0. Notably, the critical factor in selecting our growth conditions and the used mutant was mimicking infection conditions by keeping them static. The seeming discrepancy could also be related to differences in the methods used for sample preparation. As such, we used a lipid extraction system, whereas Berndt and colleagues used an extraction system optimized for species with medium polarity. Further to the phospholipid remodeling, our unbiased lipidomics analysis suggested alterations in the metabolism of terpenoid-quinones. To better understand the changes in EPEC isoprenoid metabolism following T3SS activation, we first mined a database of gene expression alterations in csrA E. coli mutant (24). The data mined implied upregulation of the undecaprenyl branch of the terpenoid quinone pathway in the $\operatorname{csr}$ A mutant (Fig. S4). As trancriptome datasets refer to wild type EPEC and $\Delta c s r A$ mutants, cultivated under different 
growth conditions than the ones used for our analyses, we assume that changes in CsrA expression may regulate lipid metabolism in E. coli spp., and in particular in EPEC. Whether and how the observed shifts in lipid metabolism contribute to host colonization are key questions that remain to be addressed in future studies. Moreover, following up on our findings in bacteria in vivo will be important to establish their possible clinical implications. Bacteria use terpenoids like undecaprenyl phosphate (Und-P) as lipid carriers to assemble numerous glycan polymers that comprise the bacterial envelope. In E. coli, Und-P is required for synthesizing peptidoglycan, Oantigen, and exopolysaccharides (29). Importantly, idi removal suppressed mutation in undecaprenyl pyrophosphate synthase (important for synthesizing undecaprenyl phosphate) (30). Our finding that the expression of both idi and ispU is higher in the sepD mutant and in the overexpressing CesT strains, suggests that EPEC attachment to the host cell may remodel its envelope structure. This notion is supported by the considerable increase in $\mathrm{O}$-antigen levels in the envelope of sepD and in the over-expressing CesT strains, and by the higher susceptibility to arge-scaffold antibiotics as vancomycin.

Our lipidomics analysis points to a considerable increase in the abundance of LysoPLs, under conditions that mimic EPEC attachment. Interestingly, LysoPLs produced by host intestinal cells induce a cAMP-dependent signaling pathway in infecting Salmonella, resulting in the production and secretion of active flagellin (31). Thus, it would be interesting to test if bacteria might use self-produced LysoPLs as a second messenger. EPEC may also produce LysoPAs to manipulate host signaling since LysoPA is a potent agonist of a family of five $\mathrm{G}$ protein-coupled receptors (GPCRs) associated with $\mathrm{G}_{\mathrm{i}}, \mathrm{G}_{12 / 13}, \mathrm{G}_{\mathrm{q}}$, and $\mathrm{G}_{\mathrm{s}}$, which activate a plethora of downstream signaling pathways including PI3K, Ras, Rho, PLC and adenylate cyclase (32-37). Moreover, the interaction of LysoPAs with GPCRs inhibits pro-inflammatory responses induced by lipopolysaccharide (LPS) $(33-35,38)$. Given the above, it is possible that LysoPL and LysoPA production and secretion may contribute to the capacity of EPEC to evade the host immune response.

In conclusion, our work provides a comprehensive view of the influence of T3SS activation and the subsequent CsrA repression on lipid metabolism and membrane biogenesis in EPEC. Notably, this study provides the community with extensive and rich resources that lay the foundation for 
follow up studies. Our data indicate that the T3SS controls the production of key components involved in the biogenesis of the bacterial cell envelope, and influences cell size, cell surface charge, and its membrane permeability. They point to a remodeling of the EPEC lipidome upon host attachment and provide a much wider view of the metabolic aspects of the bacterial switch from the planktonic to the cell-adherent lifestyle. 


\section{Methods}

\section{Bacteria cultures}

Bacterial strains and plasmids used are presented in Table S1 and primers in Table S2. All bacterial strains are isogenic. Bacteria were grown in Luria-Bertani (LB) broth supplemented, when needed for subculture, with ampicillin $(100 \mu \mathrm{g} / \mathrm{mL})$, streptomycin $(50 \mu \mathrm{g} / \mathrm{mL})$, chloramphenicol $(25 \mu \mathrm{g} / \mathrm{mL})$, kanamycin (Kan, $30 \mu \mathrm{g} / \mathrm{mL})$, or tetracycline $(10 \mu \mathrm{g} / \mathrm{mL})$. For lipid extraction EPEC were grown statically at $37^{\circ} \mathrm{C}$ overnight in $\mathrm{LB}$ medium with respective antibiotics. The overnight grown bacterial cultures were then diluted 1:50 using high glucose Dulbecco's modified Eagle medium (DMEM; Biological Industries) lacking pyruvate and glutamate, and statically grown at $37^{\circ} \mathrm{C}$. To express CesT in strains containing the pCesT plasmid, $0.05 \mathrm{mM}$ IPTG was added 3 hours after culturing in DMEM. Bacterial growth was extended to $0.6 \mathrm{Abs}$ at $\mathrm{OD}_{600}$. The cells were then centrifuged $\left(5000 \mathrm{~g}\right.$ for 10 minutes at $\left.4^{\circ} \mathrm{C}\right)$, washed twice with $20 \mathrm{~mL}$ of cold phosphate-buffered saline (PBS), and pellets were immediately snap-frozen in liquid nitrogen.

\section{Cloning, GFP fusion, and bacterial strain construction}

For GFP fusion, bacteria were electroporated with pKD46 plasmid harboring $\lambda$ Red genes $(\gamma, \beta$, and Exo) (39). The tet-sacB cassette (40) was introduced downstream to the desired gene of interest and replaced with GFP insertion. The primer sequence for GFP fusions is given as Table S2.GFP fusions were verified using PCR with flanking primers. Primers for qRT-PCR confirmation of GFP fusions are shown in Table S3. For overexpression of CesT levels, the pKD46 plasmids were cured, and pSA10 plasmid containing CesT was electroporated. Overexpression of CesT was induced upon IPTG induction.

\section{Solvents for lipidomics analysis}

Acetonitrile, methanol (both ultra-LC-MS grade), chloroform, and water (HPLC-MS grade) were supplied by JT Baker; isopropanol (HPLC-MS grade) from ChemSolute; and formic acid (HPLCMS grade) by TCI. Ammonium fluoride (>99\%) was supplied by Sigma-Aldrich. Internal standard mix - EquiSPLASH LIPIDOMIX (MS-quantitative grade) was obtained from Avanti Polar Lipids. 


\section{Sample preparation for lipidomics analyses}

Bacteria (45 mg, wet pellet) were reconstituted in $400 \mu \mathrm{L}$ of LC-MS grade water and transferred to glass tubes. Following the addition of $800 \mu \mathrm{L}$ of ice-cold methanol and internal standards (EquiSPLASH LIPIDOMIX), samples went through five cycles of 30 -sec ultra-sonication at $4^{\circ} \mathrm{C}$ for quenching and complete lysis. $400 \mu \mathrm{L}$ of cold chloroform was added, and another sequence of $30-\sec \times 5$ sonication cycles at $4^{\circ} \mathrm{C}$ was carried out. The tubes were incubated at room temperature for 30 minutes with occasional mixing (41) and then centrifuged at $770 \mathrm{~g}$ for 10 minutes at $4^{\circ} \mathrm{C}$ for phase separation. The lower chloroform phase was transferred to clean glass tubes, and the protein disk at interface was re-extracted with the same solvent system. Protein disk extracts were then pooled with the respective sample. Samples were concentrated in vacuum concentrator (126 SC210A SpeedVac; Thermo Scientific) and reconstituted in $200 \mu \mathrm{L} 95 \%$ acetonitrile, 0.1 FA. Samples were filtered through Acrodisc ${ }^{\circledR}$ PTFE membrane filters $0.2 \mu \mathrm{m}$ (Pall Corporation, USA) and transferred TO Waters ${ }^{\circledR}$ ACQUITY UPLC 700 $\mu$ l round 96 well sample plate.

\section{UPLC-MS analysis}

LC-MS analysis was carried out in a Waters Acquity UPLC H-Class (Waters, Milford, MA, USA) and Xevo X2-XS Q-ToF- High resolution, High Mass Accuracy Q-ToF (Waters, Manchester, UK) system. LC-MS runs for lipidomics analyses were performed using a UPLC CSH C18 column $(100 \mathrm{~mm} \times 2.1 \mathrm{~mm}, 1.7 \mu \mathrm{m}$; Waters $)$. The column temperature was maintained at $60^{\circ} \mathrm{C}$. The mobile phase consisted of $0.1 \%$ (vol/vol) FA in water (A), $0.1 \% \mathrm{FA}$ in acetonitrile (B), and isopropanol (C). A flow rate of $0.4 \mathrm{~mL} /$ minute was used with a linear gradient (Table S4). ESIMS was calibrated using sodium formate, and leucine enkephalin was used as the lock mass $(\mathrm{m} / \mathrm{z}$ $556.2771,200 \mathrm{pg} / \mathrm{mL}$ ) and continuously infused at $6 \mu \mathrm{L} / \mathrm{min}$. The capillary spray was maintained at $3.0 \mathrm{kV}$; the data were acquired in positive and negative mode with collision energies of 15-45 $\mathrm{eV}$ and 30-60 eV, respectively. Full $\square$ scan and $\mathrm{MS}^{\mathrm{E}}$ data acquisition were performed, ranging from 30-2000 Dalton. Argon was used as the collision gas for collision $\square$ induced dissociation. The lock mass was continuously infused at $6 \mu \mathrm{L} / \mathrm{min}$. Ammonium fluoride was used for post-column 
derivatization to improve the yields of the neutral charged lipids in the ES+ mode. MassLynx 4.1 (Waters Corporation, Milford, MA, USA) was used to control the instrument, calculate accurate masses, and mass spectral visualization.

\section{lipidomics analyses}

Progenesis QI (Nonlinear Dynamics, Newcastle, United Kingdom) was used for spectra deconvolution, alignment, and feature identification. Blank samples (solvents that went through the same sample preparation with no bacteria) were used to exclude artifactual mass features. Mass features eluted at $t>1$ minute, with minimum intensity higher than 100 , with the lowest mean abundance in the blank, and fold change over 100 from blank, were used for analysis. Following quantile normalization, multivariate tests were carried out us EZinfo 3.0 (Umetrics AB, Umea Sweden) and Metaboanalyst 4.0 (42). Full-scan and $\mathrm{MS}^{\mathrm{E}}$ mass spectra were acquired from all masses of 30-2,000 Daltons. Identification of mass features was carried out using 18 metabolite libraries compatible with Progenesis QI, as well as our internal library, based on mass accuracy $<5$ ppm, isotope pattern, fragmentation pattern, and elution time.

\section{Data mining for gene expression}

Changes in transcript levels mediated by CsrA (24) were previously reported. We mined the publicly available datasets that resulted from these studies for network-based analysis of lipid metabolism regulation by T3SS activation and CsrA inhibition. The pathways of glycerophospholipid and terpenoid quinone pathways were adapted from KEGG database and are presented and modified to allow presentation in Figure. The list of enzymes involved in E. coli lipid synthesis was extracted from the KEGG database.

\section{Determination of GFP fluorescence intensity}

The strains with GFP fusion were grown overnight in LB containing chloramphenicol at $37^{\circ} \mathrm{C}$. The overnight cultures were subcultured (1:100) in DMEM high glucose medium. IPTG was added after 3 hours, and growth continued for an additional 3 hours. The cells were washed and suspended in PBS. The fluorescence of GFP was measured at $485 \mathrm{~nm}$ excitation and $510 \mathrm{~nm}$ emission using the Spark 10M microplate reader (Tecan Trading AG, Switzerland) and normalized to their respective optical densities $\left(\mathrm{OD}_{600}\right)(43)$. 


\section{Bacterial cell size and Zeta potential measurements}

EPEC strains were subcultured in DMEM at $37^{\circ} \mathrm{C}$ to an $\mathrm{OD}_{600}$ of 0.6 . Bacteria were loaded on polylysine-coated glass slides; a coverslip was mounted on top, and the cells were visualized under a phase-contrast Axio Observer Z1 microscope (Zeiss, Germany). System control and image processing were performed with Zen pro-2012 (Zeiss, Germany). Bacterial cell size was measured using Image $\mathbf{J}$ software.

Zeta potential was measured as follows: Bacteria were centrifuged for 3 minutes at 16,000 $\mathrm{g}$ at $4^{\circ} \mathrm{C}$, washed and reconstituted with $1 \mathrm{~mL}$ of LCMS grade water. Zeta potential was determined using Zetasizer Nano ZS (Malvern Instruments, UK) at $25^{\circ} \mathrm{C}$. Zeta potential was calculated using the Smoluchowski model. Data acquisition from 15 events was recorded for every sample, and the average Zeta potential was determined from four replicates (44).

\section{Membrane staining}

For staining bacterial membranes, static cultures of EPEC strains were subcultured in DMEM at $37^{\circ} \mathrm{C}$ to an $\mathrm{OD}_{600}$ of 0.6 . The cells were washed with PBS, treated with $1 \mathrm{mg} / \mathrm{mL}$ FM4-64 (Molecular Probes, Invitrogen), and spotted on poly-L-lysine coated coverslips. Bacteria were visualized in an Eclipse Ti microscope (Nikon, Japan), equipped with a CoolSnap HQII camera (Photometrics, Roper Scientific, USA). System control, image processing, and fluorescence intensity measurement were performed with NIS Elements AR 4.3 (Nikon, Japan).

\section{LPS O-antigen Quantification}

For staining bacterial O-antigen, static cultures of EPEC strains were subcultured in DMEM at $37^{\circ} \mathrm{C}$ to an $\mathrm{OD}_{600}$ of 0.6 . Bacteria were washed with PBS and spotted onto poly-L-Lysine coated coverslips. Bacteria on the coverslips were then fixed with $2 \%$ paraformaldehyde and $0.01 \%$ glutaraldehyde in sodium cacodylate buffer $(0.1 \mathrm{M}, \mathrm{pH} 7.2)$ for 15 minutes at $25^{\natural} \mathrm{C}$. Subsequently, coverslips were washed 3 times in PBS, incubated for 30 minutes with 2\% BSA and then with rabbit anti-O127 antibody (1:500, 2\% BSA), for 1 hour at $25^{\square} \mathrm{C}$. Coverslips were washed 3 times with PBS and incubated for 1 hour at $25^{\square} \mathrm{C}$ with Alexa 488 conjugated goat anti-rabbit antibody (1:1000). Coverslips were then washed 3 times with PBS and fixed with $2.5 \%$ glutaraldehyde in 
sodium cacodylate buffer $(0.1 \mathrm{M}, \mathrm{pH} 7.2)$ for 15 minutes at $25^{\square} \mathrm{C}$. Bacteria were visualized by Eclipse Ti microscope (Nikon, Japan), equipped with CoolSnap HQII camera (Photometrics, Roper Scientific, USA). System control, image processing and fluorescence intensity measurement were performed with NIS Elements AR 4.3 (Nikon, Japan).

\section{LPS Extraction and O-antigen quantificaition}

For LPS extraction overnight grown cultures in Luria Broth (LB), supplemented with antibiotics (see Bacteria cultures) were subcultured in $\mathrm{DMEM}$ at $37^{\circ} \mathrm{C}$ to an $\mathrm{OD}_{600}$ of 0.6 . The cells were centrifuged at $10,000 \mathrm{x} \mathrm{g}$ for 10 minutes and the pellet was collected. The pellets were reconstituted in $200 \mu \mathrm{L}$ Laemelli buffer with bromophenol blue dye, and kept at $95^{\square} \mathrm{c}$ for 15 minutes and allowed to cool at room temperature for 15 minutes. $10 \mu \mathrm{L}$ of Proteinase $\mathrm{K}$ solution $(10 \mathrm{mg} / \mathrm{mL})$ was added to the samples and incubated at $59{ }^{\natural} \mathrm{C}$ for 3 hours. $200 \mu \mathrm{L}$ of ice cold water saturated phenol was added to the samples. The samples were vortexed for approximately 5 to 10 seconds, and incubated at $65^{\square} \mathrm{C}$ for 15 minutes with occasional vortexing. After cooling to room temperature, $1 \mathrm{~mL}$ of diethyl ether was added to each sample, followed by vortex (5-10 seconds). The samples were centrifuged (13000 g, 10 minutes). The lower blue layer was carefully removed, and samples were run on $12 \%$ SDS-polyacrylamide gels (MiniPROTEAN@ TGX Stain-Free ${ }^{\mathrm{TM}}$ ). The bands were transferred to $0.2 \mu \mathrm{M}$ Nitrocellulose membrane (Trans Blot Turbo, Biorad laboratories, USA). The blot was blocked with BSA (Bovine serum Albumin) and skim milk $(0.6 \%)$ in TBS overnight at $4^{\square} \mathrm{C}$. The membrane was then incubated with Rabbit-AntiO127 antibody (1:1000) for 1 hour at room temperature, washed 3 times with TBST and incubated with Anti Rabbit IgG (Whole molecule )-AP antibody (1:10000) for one hour. The membrane was then washed with TBST two times. Finally the membrane was washed with AP buffer (100 mM Tris- $\mathrm{HCl}$ [pH 9.0], $150 \mathrm{mM} \mathrm{NaCl}, 1 \mathrm{mM} \mathrm{MgCl}_{2}$ ). The membrane was visualized using GeldocTm EZimagin (Biorad USA) with transwhite background.

\section{Serum Bactericidal assay}

Overnight grown EPEC strains were subcultured in DMEM medium in 96 well transparent flatbottom plate. Human Serum samples were diluted from 1:50-1:6250 dilutions and incubated with EPEC strains at $37^{\circledR} \mathrm{C}$. Serum samples were heat inactivated at $56^{\circledR} \mathrm{C}$. The growth rate was 
monitored at $600 \mathrm{~nm}$ for 12 hours using Spark $10 \mathrm{M}$ microplate reader (Tecan Trading AG, Switzerland)

\section{Vancomycin Bactericidal assay}

Overnight grown EPEC strains were subcultured in DMEM medium in 96 well transparent flatbottom plate. Vancomycin (Sigma Aldrich, Louis ,MO, USA; 0, 3, 10, 30, $100 \mu \mathrm{M}$ ) was incubated with EPEC strains at $37^{\llbracket} \mathrm{C}$. The growth rate was monitored at $600 \mathrm{~nm}$ for 12 hours using Spark 10M microplate reader (Tecan Trading AG, Switzerland).

\section{Competing interests}

We have no competing interests to report. 


\section{References}

1. Nataro JP, Kaper JB. 1998. Diarrheagenic Escherichia coli. Clin Microbiol Rev 11:142-201.

2. Elliott SJ, Wainwright LA, McDaniel TK, Jarvis KG, Deng YK, Lai LC, McNamara BP, Donnenberg MS, Kaper JB. 1998. The complete sequence of the locus of enterocyte effacement (LEE) from enteropathogenic Escherichia coli E2348/69. Mol Microbiol 28:1-4.

3. Garmendia J, Frankel G, Crepin VF. 2005. Enteropathogenic and enterohemorrhagic Escherichia coli infections: translocation, translocation, translocation. Infect Immun 73:2573-85.

4. Gaytan MO, Martinez-Santos VI, Soto E, Gonzalez-Pedrajo B. 2016. Type Three Secretion System in Attaching and Effacing Pathogens. Front Cell Infect Microbiol 6:129.

5. Mellies JL, Elliott SJ, Sperandio V, Donnenberg MS, Kaper JB. 1999. The Per regulon of enteropathogenic Escherichia coli : identification of a regulatory cascade and a novel transcriptional activator, the locus of enterocyte effacement (LEE)-encoded regulator (Ler). Mol Microbiol 33:296-306.

6. Sanchez-SanMartin C, Bustamante VH, Calva E, Puente JL. 2001. Transcriptional regulation of the orf19 gene and the tir-cesT-eae operon of enteropathogenic Escherichia coli. J Bacteriol 183:2823-33.

7. Kenny B, DeVinney R, Stein M, Reinscheid DJ, Frey EA, Finlay BB. 1997. Enteropathogenic E. coli (EPEC) transfers its receptor for intimate adherence into mammalian cells. Cell 91:511-20.

8. Mills E, Baruch K, Aviv G, Nitzan M, Rosenshine I. 2013. Dynamics of the type III secretion system activity of enteropathogenic Escherichia coli. mBio 4.

9. Little DJ, Coombes BK. 2018. Molecular basis for CesT recognition of type III secretion effectors in enteropathogenic Escherichia coli. PLoS Pathog 14:e1007224.

10. Runte CS, Jain U, Getz U, Secord S, Kuwae A, Abe A, LeBlanc JJ, Stadnyk AW, Kaper JB, Hansen AM, Thomas NA. 2018. Tandem tyrosine phosphosites in the Enteropathogenic Escherichia coli chaperone CesT are required for differential type III effector translocation and virulence. Mol Microbiol 108:536-550.

11. Lai Y, Rosenshine I, Leong JM, Frankel G. 2013. Intimate host attachment: enteropathogenic and enterohaemorrhagic Escherichia coli. Cell Microbiol 15:1796-808.

12. Login FH, Jensen HH, Pedersen GA, Amieva MR, Nejsum LN. 2018. The soluble extracellular domain of E-cadherin interferes with EPEC adherence via interaction with the Tir:intimin complex. FASEB J doi:10.1096/fj.201800651:fj201800651.

13. Katsowich N, Elbaz N, Pal RR, Mills E, Kobi S, Kahan T, Rosenshine I. 2017. Host cell attachment elicits posttranscriptional regulation in infecting enteropathogenic bacteria. Science 355:735-739.

14. Mills E, Baruch K, Charpentier X, Kobi S, Rosenshine I. 2008. Real-time analysis of effector translocation by the type III secretion system of enteropathogenic Escherichia coli. Cell Host Microbe 3:104-13.

15. Goddard PJ, Sanchez-Garrido J, Slater SL, Kalyan M, Ruano-Gallego D, Marches O, Fernandez LA, Frankel G, Shenoy AR. 2019. Enteropathogenic Escherichia coli Stimulates Effector-Driven Rapid Caspase-4 Activation in Human Macrophages. Cell Rep 27:1008-1017 e6.

16. Ye F, Yang FL, Yu RJ, Lin X, Qi JX, Chen ZJ, Cao Y, Wei YQ, Gao GF, Lu GW. 2018. Molecular basis of binding between the global post-transcriptional regulator CsrA and the T3SS chaperone CesT. Nature Communications 9.

17. Potts AH, Vakulskas CA, Pannuri A, Yakhnin H, Babitzke P, Romeo T. 2017. Global role of the bacterial post-transcriptional regulator CsrA revealed by integrated transcriptomics. Nat Commun 8:1596. 
18. Romeo T, Gong M, Liu MY, Brun-Zinkernagel AM. 1993. Identification and molecular characterization of csrA, a pleiotropic gene from Escherichia coli that affects glycogen biosynthesis, gluconeogenesis, cell size, and surface properties. J Bacteriol 175:4744-55.

19. Sowa SW, Gelderman G, Leistra AN, Buvanendiran A, Lipp S, Pitaktong A, Vakulskas CA, Romeo T, Baldea M, Contreras LM. 2017. Integrative FourD omics approach profiles the target network of the carbon storage regulatory system. Nucleic Acids Res 45:1673-1686.

20. McKee AE, Rutherford BJ, Chivian DC, Baidoo EK, Juminaga D, Kuo D, Benke PI, Dietrich JA, Ma SM, Arkin AP, Petzold CJ, Adams PD, Keasling JD, Chhabra SR. 2012. Manipulation of the carbon storage regulator system for metabolite remodeling and biofuel production in Escherichia coli. Microb Cell Fact 11:79.

21. Sabnis NA, Yang H, Romeo T. 1995. Pleiotropic regulation of central carbohydrate metabolism in Escherichia coli via the gene csrA. J Biol Chem 270:29096-104.

22. Suzuki K, Wang X, Weilbacher T, Pernestig AK, Melefors O, Georgellis D, Babitzke P, Romeo T. 2002. Regulatory circuitry of the CsrA/CsrB and BarA/UvrY systems of Escherichia coli. J Bacteriol 184:5130-40.

23. Deng W, Yu HB, Li Y, Finlay BB. 2015. SepD/SepL-dependent secretion signals of the type III secretion system translocator proteins in enteropathogenic Escherichia coli. J Bacteriol 197:126375.

24. Berndt V, Beckstette M, Volk M, Dersch P, Bronstrup M. 2019. Metabolome and transcriptomewide effects of the carbon storage regulator $A$ in enteropathogenic Escherichia coli. Sci Rep 9:138.

25. Morin M, Ropers D, Letisse F, Laguerre S, Portais JC, Cocaign-Bousquet M, Enjalbert B. 2016. The post-transcriptional regulatory system CSR controls the balance of metabolic pools in upper glycolysis of Escherichia coli. Mol Microbiol 100:686-700.

26. Taniguchi Y, Choi PJ, Li GW, Chen H, Babu M, Hearn J, Emili A, Xie XS. 2010. Quantifying E. coli proteome and transcriptome with single-molecule sensitivity in single cells. Science 329:533-8.

27. Muheim C, Gotzke H, Eriksson AU, Lindberg S, Lauritsen I, Norholm MHH, Daley DO. 2017. Increasing the permeability of Escherichia coli using MAC13243. Sci Rep 7:17629.

28. Revelles O, Millard P, Nougayrede JP, Dobrindt U, Oswald E, Letisse F, Portais JC. 2013. The carbon storage regulator (Csr) system exerts a nutrient-specific control over central metabolism in Escherichia coli strain Nissle 1917. PLoS One 8:e66386.

29. Jorgenson MA, Young KD. 2016. Interrupting Biosynthesis of O Antigen or the Lipopolysaccharide Core Produces Morphological Defects in Escherichia coli by Sequestering Undecaprenyl Phosphate. J Bacteriol 198:3070-3079.

30. MacCain WJ, Kannan S, Jameel DZ, Troutman JM, Young KD. 2018. A Defective Undecaprenyl Pyrophosphate Synthase Induces Growth and Morphological Defects That Are Suppressed by Mutations in the Isoprenoid Pathway of Escherichia coli. J Bacteriol 200.

31. Subramanian N, Qadri A. 2006. Lysophospholipid sensing triggers secretion of flagellin from pathogenic salmonella. Nat Immunol 7:583-9.

32. Chien HY, Lu CS, Chuang KH, Kao PH, Wu YL. 2015. Attenuation of LPS-induced cyclooxygenase-2 and inducible NO synthase expression by lysophosphatidic acid in macrophages. Innate Immun 21:635-46.

33. Zhao J, He D, Su Y, Berdyshev E, Chun J, Natarajan V, Zhao Y. 2011. Lysophosphatidic acid receptor 1 modulates lipopolysaccharide-induced inflammation in alveolar epithelial cells and murine lungs. Am J Physiol Lung Cell Mol Physiol 301:L547-56.

34. Fan H, Zingarelli B, Harris V, Tempel GE, Halushka PV, Cook JA. 2008. Lysophosphatidic acid inhibits bacterial endotoxin-induced pro-inflammatory response: potential anti-inflammatory signaling pathways. Mol Med 14:422-8. 
35. Ciesielska A, Hromada-Judycka A, Ziemlinska E, Kwiatkowska K. 2019. Lysophosphatidic acid upregulates IL-10 production to inhibit TNF-alpha synthesis in Mvarphis stimulated with LPS. J Leukoc Biol 106:1285-1301.

36. Lin S, Han Y, Jenkin K, Lee SJ, Sasaki M, Klapproth JM, He P, Yun CC. 2018. Lysophosphatidic Acid Receptor 1 Is Important for Intestinal Epithelial Barrier Function and Susceptibility to Colitis. Am J Pathol 188:353-366.

37. Wiedmaier N, Muller S, Koberle M, Manncke B, Krejci J, Autenrieth IB, Bohn E. 2008. Bacteria induce CTGF and CYR61 expression in epithelial cells in a lysophosphatidic acid receptordependent manner. Int J Med Microbiol 298:231-43.

38. Tsai HC, Han MH. 2016. Sphingosine-1-Phosphate (S1P) and S1P Signaling Pathway: Therapeutic Targets in Autoimmunity and Inflammation. Drugs 76:1067-79.

39. Datsenko KA, Wanner BL. 2000. One-step inactivation of chromosomal genes in Escherichia coli K12 using PCR products. Proc Natl Acad Sci U S A 97:6640-5.

40. Li XT, Thomason LC, Sawitzke JA, Costantino N, Court DL. 2013. Positive and negative selection using the tet $A-s a c B$ cassette: recombineering and P1 transduction in Escherichia coli. Nucleic Acids Res 41:e204.

41. Rowlett VW, Mallampalli V, Karlstaedt A, Dowhan W, Taegtmeyer H, Margolin W, Vitrac H. 2017. Impact of Membrane Phospholipid Alterations in Escherichia coli on Cellular Function and Bacterial Stress Adaptation. J Bacteriol 199.

42. Chong J, Soufan O, Li C, Caraus I, Li S, Bourque G, Wishart DS, Xia J. 2018. MetaboAnalyst 4.0: towards more transparent and integrative metabolomics analysis. Nucleic Acids Res 46:W486W494.

43. Elbaz N, Socol Y, Katsowich N, Rosenshine I. 2019. Control of Type III Secretion System Effector/Chaperone Ratio Fosters Pathogen Adaptation to Host-Adherent Lifestyle. mBio 10.

44. Wyness AJ, Paterson DM, Defew EC, Stutter MI, Avery LM. 2018. The role of zeta potential in the adhesion of E. coli to suspended intertidal sediments. Water Res 142:159-166. 
bioRxiv preprint doi: https://doi.org/10.1101/2020.12.17.423234; this version posted June 27, 2021. The copyright holder for this preprint (which was not certified by peer review) is the author/funder, who has granted bioRxiv a license to display the preprint in perpetuity. It is made available under aCC-BY-NC 4.0 International license. 